\title{
Divergence of the Grüneisen Parameter and Magnetocaloric Effect at Heavy Fermion Quantum Critical Points
}

\author{
P. Gegenwart • Y. Tokiwa • J.G. Donath • \\ R. Küchler · C. Bergmann · H.S. Jeevan · \\ E.D. Bauer · J.L. Sarrao • C. Geibel · F. Steglich
}

Received: 11 April 2010 / Accepted: 20 June 2010 / Published online: 7 July 2010

(C) The Author(s) 2010. This article is published with open access at Springerlink.com

\begin{abstract}
At any pressure sensitive quantum critical point (QCP) the thermal expansion is more singular than the specific heat leading to a divergence of the Grüneisen parameter. For a magnetic field sensitive QCP, the complementary property is the magnetic Grüneisen ratio which equals the magnetocaloric effect. Here we use both properties to investigate magnetic QCPs in different heavy fermion (HF) metals starting from $\mathrm{CeNi}_{2} \mathrm{Ge}_{2}$. The influence of dimensionality on quantum criticality is addressed by the comparison of cubic CeIn ${ }_{3-x} \mathrm{Sn}_{x}$ with layered CeMIn ${ }_{5-x} \mathrm{Sn}_{x}$ $(\mathrm{M}=\mathrm{Co}, \mathrm{Rh})$ systems, in which $\mathrm{Sn}$ doping both acts as tuning parameter and introduces slight disorder. Near the field-tuned QCP in undoped CeCoIn 5 a crossover scale $T^{\star}$ is discovered which separates $2 \mathrm{D}$ (at $T>T^{\star}$ ) from 3D (at $T<T^{\star}$ ) quantum criticality. Disorder, introduced by Sn-doping, is found to increase $T^{\star}$, stabilizing 3D behavior. We also compare the magnetic Grüneisen ratio in the approach of the field-tuned QCP in $\mathrm{YbRh}_{2} \mathrm{Si}_{2}$ with zero-field Grüneisen parameter data on $\mathrm{YbRh}_{2}\left(\mathrm{Si}_{1-x} \mathrm{Ge}_{x}\right)_{2}(x=0, x=0.05)$. Both properties indicate quantum criticality incompatible with the predictions of the itinerant theory.
\end{abstract}

Keywords Quantum critical point · Heavy fermion systems

P. Gegenwart $(\bowtie) \cdot$ Y. Tokiwa $\cdot$ C. Bergmann · H.S. Jeevan First Physics Institute, Georg-August-University, 37077 Göttingen, Germany e-mail: pgegenw@gwdg.de

J.G. Donath · R. Küchler · C. Geibel · F. Steglich

Max-Planck-Institute for Chemical Physics of Solids, 01187 Dresden, Germany

E.D. Bauer · J.L. Sarrao

Los Alamos National Laboratory, Los Alamos, NM 87545, USA 


\section{Quantum Criticality in Heavy Fermion Metals}

Heavy fermion (HF) metals are intermetallic compounds with rare-earth (most Ce or $\mathrm{Yb}$ ) or actinide (most $\mathrm{U}$ ) elements in which the effective charge-carrier masses are highly enhanced compared to the free electron mass [1-3]. Their ground state sensitively depends on the balance between the on-site Kondo and the inter-site exchange interaction. Thus, it is easy to tune these systems by either small changes in chemical composition, the application of moderate pressure or the application of magnetic fields. By varying such control parameters, long-range antiferromagnetic (AF) ordering could often be continuously suppressed towards a quantum critical point (QCP) $T_{\mathrm{N}} \rightarrow 0[4,5]$. While HF systems are prototype systems for the realization of quantum criticality, their theoretical description near the QCP is subject of continued interest. The main complication arises from the fact that heavy quasiparticles (QP) are composite objects formed by f-electrons with conduction electron admixture [6]. The crucial question is whether these heavy quasiparticles stay intact at the QCP or decompose due to the destruction of the Kondo singlet formation. In the former case the well-developed Hertz-Millis-Moriya theory is applicable. This is an itinerant description of quantum criticality, implying the AF order to be of spindensity-wave (SDW) type [7-9]. The observation through inelastic neutron scattering (INS) that the AF correlations at the QCP in $\mathrm{CeCu}_{6-x} \mathrm{Au}_{x}$ are of local character [10], together with the stronger than logarithmic mass divergence in the approach of the QCP in $\mathrm{YbRh}_{2}\left(\mathrm{Si}_{0.95} \mathrm{Ge}_{0.05}\right)_{2}$ [11], suggest a decomposition of the heavy QP due to the breakdown of the Kondo effect, adding electronic degrees of freedom to the critical (magnetic) fluctuations [6, 12-15].

Quantum critical fluctuations result in an anomalous enhancement of the entropy near the QCP. The dimensionless Grüneisen parameter defined by $\Gamma=\frac{V_{m}}{\kappa_{T}} \frac{\beta}{C}$ where $C$ and $\beta$ denote the specific heat and the volume expansion, respectively ( $V_{m}$ : molar volume, $\kappa_{T}$ : isothermal compressibility) is an important tool to study this entropy enhancement. Whenever a system is dominated by a single energy scale $T_{0}$, e.g. the Fermi energy in a metal or the Debye energy for phonons, the free energy scales as $f\left(T / T_{0}\right)$ leading to a temperature independent $\Gamma=-\frac{\partial \log T_{0}}{\partial \log V}$, which is known as Grüneisen law [16]. While for normal metals $\Gamma \sim 1$, its absolute value is largely enhanced for HF metals $(|\Gamma| \sim 100)$ at low temperatures $[17,18]$, as a consequence of the strong volume dependence of the narrow heavy quasiparticle band.

For HF metals, the "coherence temperature", below which a Landau-Fermi-liquid (LFL) state forms, takes the role of $T_{0}$ which vanishes at the QCP. Consequently, the Grüneisen parameter will diverge analytically at any pressure-sensitive QCP. Within the itinerant theory the critical component of the Grüneisen ratio (after subtraction of non-critical contributions to specific heat and thermal expansion, see below) diverges as $\Gamma_{\mathrm{cr}} \propto T^{-\varepsilon}$ with $\varepsilon=(\nu z)^{-1}$, where $v$ and $z$ are the correlation-length exponent and the dynamic exponent, respectively [19]. In case of a magnetic-field driven QCP, similar results apply to the magnetic Grüneisen ratio $\Gamma_{\operatorname{mag}}=-\frac{(\partial M / \partial T)}{C}=\frac{1}{T}(\partial T / \partial B)_{S}$. The latter property equals the magnetocaloric effect, defined as the relative change of temperature in response to an adiabatic change of the magnetic field.

An early study of the thermal expansion and Grüneisen ratio for a system close to a QCP focussed on $\mathrm{Ce}_{1-x} \mathrm{La}_{x} \mathrm{Ru}_{2} \mathrm{Si}_{2}$ with $x=0$ and $x=0.05$ in which negative 


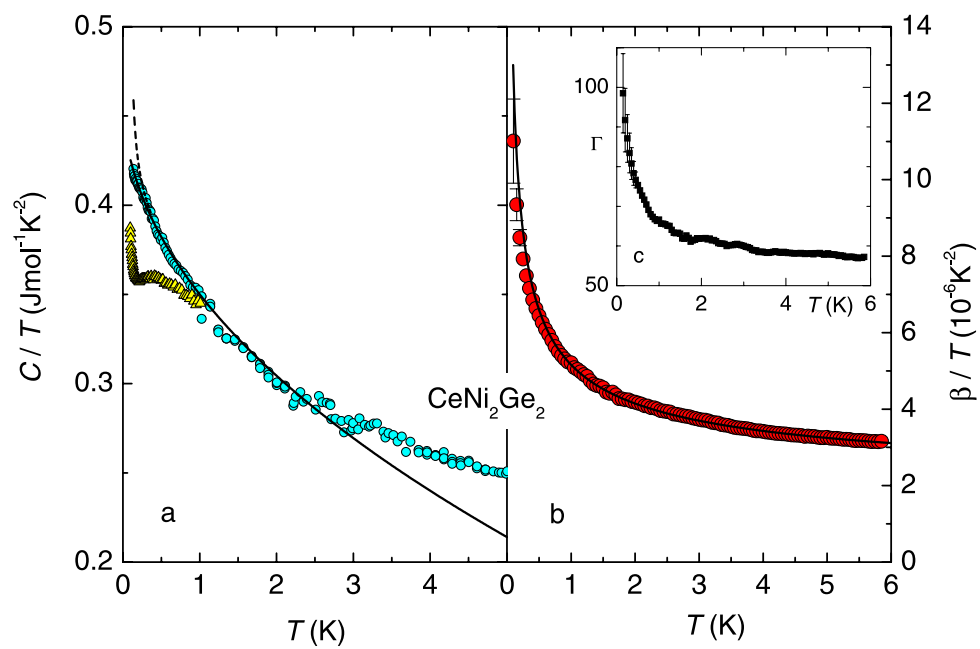

Fig. 1 (Color online) (a) Low-temperature specific heat as $C / T$ vs. $T$ for a $\mathrm{CeNi}_{2} \mathrm{Ge}_{2}$ single crystal [21]. The solid line represents a fit according to the 3D SDW scenario, i.e. $C(T) / T=\gamma_{0}-c \sqrt{T}$, with $\gamma_{0}=0.46 \mathrm{~J} / \mathrm{K}^{2}$ mol and $c=0.11 \mathrm{~J} / \mathrm{K}^{5 / 2}$ mol, where $C$ denotes the specific heat (circles) after subtraction of a nuclear contribution from the raw data (dashed line at low $T$ ). The yellow triangles show data at $B=2 \mathrm{~T}$ to illustrate the independency of the low-temperature "upturn" from the electronic contribution. (b) Corresponding volume thermal expansion of the same single crystal as $\beta / T$ vs. $T$. The line indicates $\beta(T)=a \sqrt{T}+b T$ with $a=3.5 \times 10^{-6} \mathrm{~K}^{-1.5}$ and $b=1.7 \times 10^{-6} \mathrm{~K}^{-2}$. Inset (c) displays the $T$ dependence of the Grüneisen ratio $\Gamma=\left(V_{m} / \kappa_{T}\right)(\beta / C)$. Here $V_{m}$ and $\kappa_{T}$ denote the molar volume and isothermal compressibility, respectively

chemical pressure induced by La-doping tunes the system towards an AF QCP at $x_{c} \approx 0.08$ [20]. A very large value of $\Gamma$ was obtained for $x=0.05$ which, however, was found to saturate below $0.8 \mathrm{~K}$, probably because the QCP has not exactly been reached as $T \rightarrow 0$.

A truly divergent Grüneisen ratio has first been observed for the clean HF metal $\mathrm{CeNi}_{2} \mathrm{Ge}_{2}$ [21]. Although this system remains paramagnetic down to lowest temperatures, it is located very close to a zero-field QCP as electrical resistivity measurements have found a divergence of the coefficient $A$ in $\rho(T)=\rho_{0}+A T^{2}$ for $B \rightarrow 0$ [22]. As shown in Fig. 1a, the electronic specific heat coefficient could be described by $C(T) / T=\gamma_{0}-c \sqrt{T}$ compatible with the itinerant theory for a 3D AF QCP. Here, we have assumed that the low-temperature upturn, described by a Schottky anomaly, is not part of the quantum critical contribution, since it remains unchanged at a field of $2 \mathrm{~T}$ (see the yellow circles) that tunes the system away from the QCP towards the LFL regime. The volume thermal expansion coefficient $\beta(T) / T=a_{0}+a_{1} / \sqrt{T}$ is far more singular than the specific heat coefficient and in perfect agreement with the predictions of the itinerant theory for a 3D AF QCP. The Grüneisen parameter $\Gamma(T)$ displays a stronger than logarithmic divergence, providing evidence for a QCP, cf. Fig. 1c. In order to analyze the critical Grüneisen ratio, the normal contributions to specific heat and thermal expansion must be subtracted, leading to $C_{\mathrm{cr}} \propto-T^{3 / 2}$ (cf. Fig. 2a), $\beta_{\text {cr }} \propto \sqrt{T}$ and thus $\Gamma_{c r} \propto-T^{-1}$, as shown in Fig. 2b [21]. Since for an 


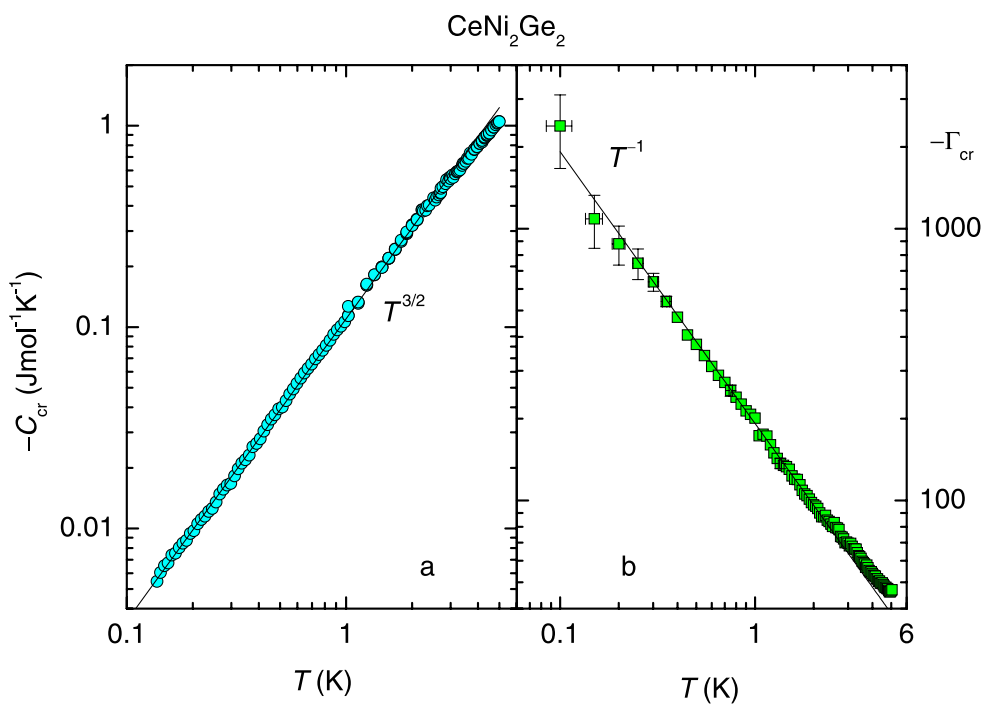

Fig. 2 (Color online) (a) Log-log plot of the critical specific heat $C_{\mathrm{cr}}=C(T)-\gamma_{0} T$ for $C(T)$ as displayed in Fig. 1a. The line indicates $-C_{\mathrm{cr}}=c T^{3 / 2}$. (b) Log-log plot of the critical Grüneisen ratio $\Gamma_{\mathrm{cr}}=\left(V_{m o l} / \kappa_{T}\right)\left(\beta_{\mathrm{cr}} / C_{\mathrm{cr}}\right)$ with $\beta_{\mathrm{cr}}=\beta(T)-b T$ (cf. Fig. 1b). The line displays a $1 / T$ divergence [21]

AF QCP $v=1 / 2$ and $z=2$, this temperature dependence agrees perfectly with the prediction of the itinerant theory [19].

A volume expansion, induced by the partial substitution of $\mathrm{Ni}$ with larger $\mathrm{Pd}$ has been shown to induce long-range $\mathrm{AF}$ order in $\mathrm{Ce}\left(\mathrm{Ni}_{1-x} \mathrm{Pd}_{x}\right)_{2} \mathrm{Ge}_{2}$ polycrystals, e.g. $T_{N}=2 \mathrm{~K}$ for $x=0.2$ [23]. Very recently, we have started the single crystal growth of undoped [24] and Pd-doped [25] $\mathrm{CeNi}_{2} \mathrm{Ge}_{2}$ using the floating zone technique in a four-mirror optical furnace under high-purity argon atmosphere. Here, we discuss first yet unpublished data of the low-temperature specific heat and magnetic Grüneisen ratio of a $x=0.065$ single crystal. The latter property has been determined by using a quasi-adiabatic field-modulation technique with amplitude of about $5 \mathrm{mT}$ at a frequency of $0.1 \mathrm{~Hz}$ [26]. As shown in Fig. 3, the low- $T$ specific heat coefficient has increased by more than a factor of two compared to undoped $\mathrm{CeNi}_{2} \mathrm{Ge}_{2}$. This indicates a reduction of the Kondo temperature by negative chemical pressure and a substantial increase of fluctuations as the system is driven closer towards the QCP. Similar as for the undoped system, NFL behavior is suppressed by the application of small magnetic fields, indicating the vicinity to a zero-field QCP. This is also compatible with measurements of the magnetocaloric effect $\Gamma_{\text {mag }}(T)$, which indicate a strong divergence at low magnetic fields. Further experiments, in particular measurements of the thermal expansion, are required to investigate the nature of the substitution-driven QCP in $\mathrm{Ce}\left(\mathrm{Ni}_{1-x} \mathrm{Pd}_{x}\right)_{2} \mathrm{Ge}_{2}$.

In case of the HF system $\mathrm{CeCu}_{6-x} \mathrm{Ag}_{x}$, which displays an AF QCP close to $x=0.2$, the thermal expansion coefficient and Grüneisen ratio could not be described within the itinerant theory [27]. In this system $\Gamma_{c r}(T)$ diverges only logarithmically, much too weak compared to the expected $1 / T$ dependence. In the related system 


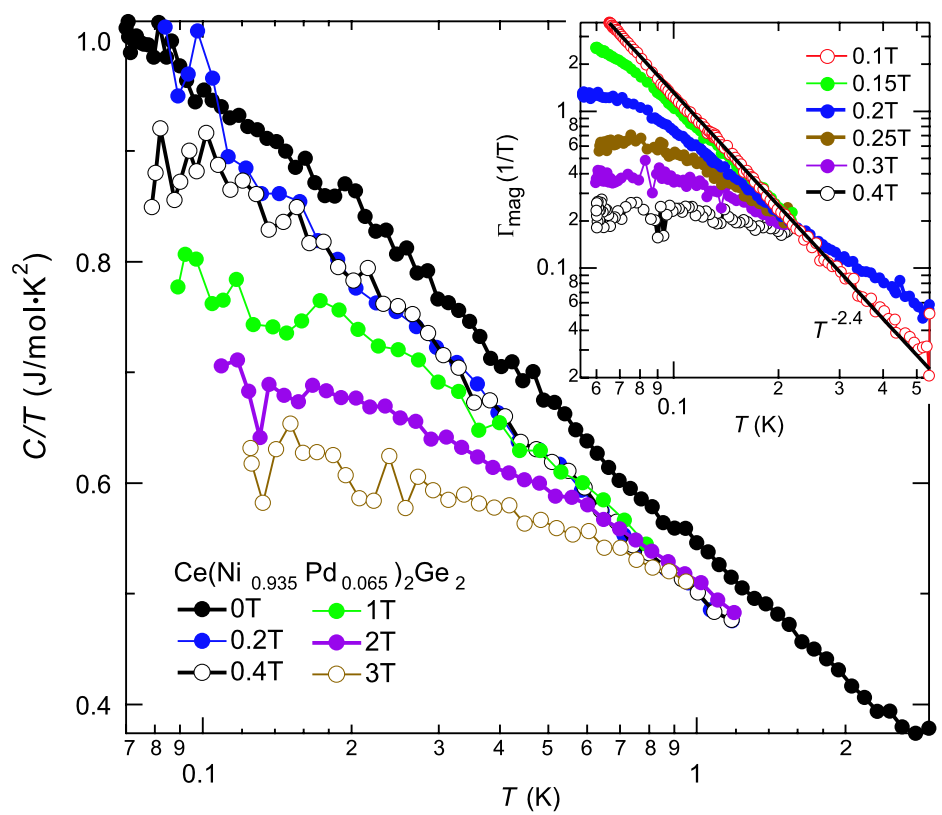

Fig. 3 (Color online) Low-temperature specific heat as $C / T$ vs. $\log T$ of single crystalline $\mathrm{Ce}\left(\mathrm{Ni}_{0.935} \mathrm{Pd}_{0.065}\right)_{2} \mathrm{Ge}_{2}$ at various different magnetic fields applied along the a-direction. The inset displays the magnetic Grüneisen parameter $\Gamma_{\text {mag }}=T^{-1}(d T / d B)_{S}$, which has been determined by adiabatic magnetocaloric effect measurements [25]

$\mathrm{CeCu}_{5.9} \mathrm{Au}_{0.1}$ evidence for quasi-2D quantum critical fluctuations has been found in INS experiments [28]. A 2D AF QCP of itinerant nature could however be excluded from the observation of energy-over-temperature scaling in dynamical susceptibility with an anomalous fractional exponent, virtually independent of wave vector [10]. This observation has led to the development of the locally-critical QCP scenario which considers a breakdown of the Kondo effect at the QCP [12]. The experiments on $\mathrm{CeCu}_{5.9} \mathrm{Au}_{0.1}$ suggest that the quasi $2 \mathrm{D}$ magnetic fluctuations are crucial for the occurrence of such an unconventional QCP. Theoretically, it has also been shown that strong geometrical frustration may act as competing mechanism against Kondosinglet formation, since it is leading to spin-liquid formation [13]. Therefore, it is of particular interest to study the influence of the parameter "dimensionality" on quantum criticality in HF systems. This is addressed in the following section. Section 3 considers the role of different tuning parameters by a comparison of the Grüneisen parameter and the magnetocaloric effect in chemically substituted and magnetic field tuned $\mathrm{YbRh}_{2} \mathrm{Si}_{2}$, respectively. This system has been in the focus of attention for several years because it shows divergences of the QP effective mass and QP-QP scattering cross-section that are incompatible with the itinerant theory [11, 29]. Furthermore, a drastic change of the Hall-coefficient [30] upon tuning through the quantum critical point (QCP), which is also accompanied by signatures in thermodynamic and magnetic properties [31] has been found which suggests a Kondo breakdown QCP in this system. 


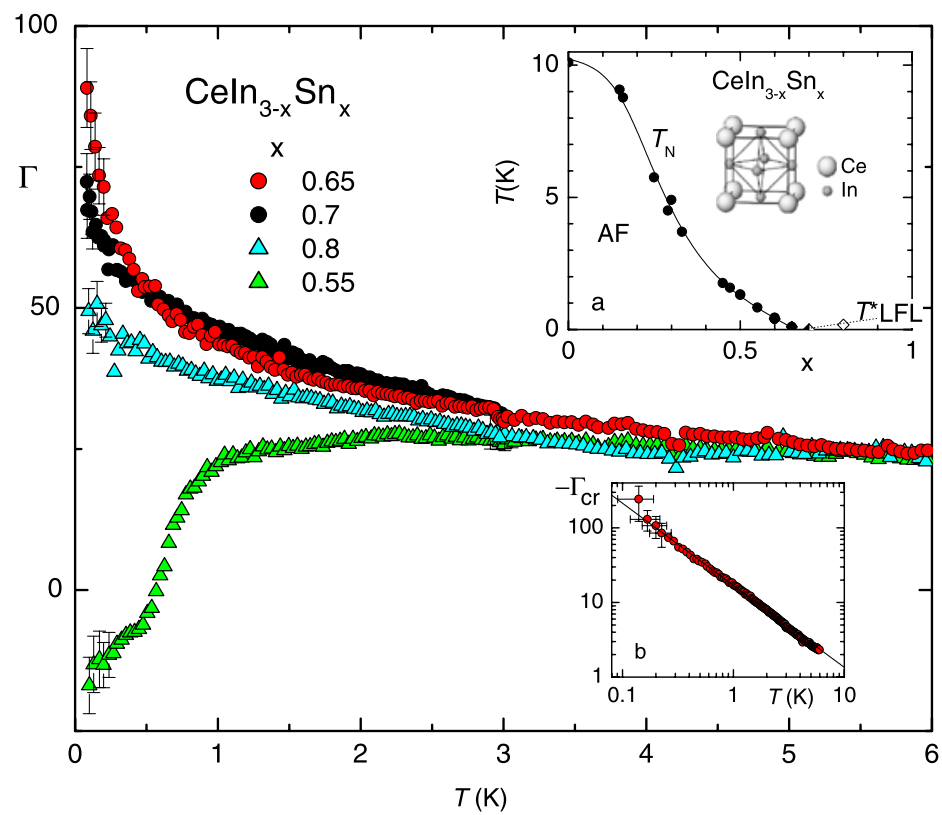

Fig. 4 (Color online) Temperature dependence of the Grüneisen parameter of several CeIn ${ }_{3-x} \operatorname{Sn}_{x}$ single crystals [32]. Inset (a) shows the magnetic phase diagram for cubic CeIn ${ }_{3-x} \operatorname{Sn}_{x}$. Inset (b) displays the temperature dependence of the critical Grüneisen ratio (after subtraction of non-critical contributions to thermal expansion and specific heat) of $\mathrm{CeIn}_{2.35} \mathrm{Sn}_{0.65}$ on double-log scale

\section{Comparison of Cubic and Layered Heavy-Fermion Systems}

The HF system CeIn ${ }_{3-x} \mathrm{Sn}_{x}$, which crystallizes in the cubic $\mathrm{Cu}_{3} \mathrm{Au}$ structure is suitable for a comparative study of the influence of dimensionality on quantum criticality, since here low-dimensional fluctuations could be ruled out due to the cubic point symmetry of the Ce-atoms. The inset a of Fig. 4 displays the magnetic phase diagram as determined by various thermodynamic and magnetic experiments [32]. Whereas pressure experiments on $\mathrm{CeIn}_{3}$ have revealed an abrupt disappearance of AF order at $p_{c}=2.6 \mathrm{GPa}$ with superconductivity $(\mathrm{SC})$ at $0.2 \mathrm{~K}$ in a very narrow pressure regime close to $p_{c}$ [33], $T_{\mathrm{N}}(x)$ evolves continuously in $\mathrm{CeIn}_{3-x} \mathrm{Sn}_{x}$. The partial substitution of In with Sn leads to an increase of the conduction electron density of states which enhances the Kondo temperature and thereby weakens the long-range AF order [34]. In this system $T_{\mathrm{N}}$ could be traced down to $0.1 \mathrm{~K}$ [32]. A further advantage for the study of quantum criticality in this system is that there appears no SC phase around the QCP. Therefore, quantum criticality could be accessed down to lowest temperatures and a detailed study of the thermal expansion, specific heat and Grüneisen parameter has been performed on well characterized single-crystals [32]. The obtained data for $\Gamma(T)$ are displayed in Fig. 4. The AF phase transition for $x=0.55$ at $T_{\mathrm{N}} \approx 0.7 \mathrm{~K}$ is manifested in a smeared discontinuity. Furthermore, the entropy accumulation near the phase transition leads to a sign change of the Grüneisen parameter, as theoretically predicted [35]. Since $\Gamma=-\frac{\partial \log T_{0}}{\partial \log V}=\frac{1}{\kappa_{T} \cdot T_{0}} \cdot \frac{d T_{0}}{d p}$, the sign of 
the Grüneisen parameter reflects the pressure dependence of the characteristic energy scale $T_{0}$ of the system. For Ce-based HF systems in the paramagnetic state, both the Kondo and coherence temperature increase with pressure leading to $\Gamma>0$. On the other hand, the negative sign in the AF state reflects the negative pressure dependence of the Néel temperature close to the QCP. The extrapolation of $T_{\mathrm{N}}(x) \rightarrow 0$ leads to $x_{c}=0.67 \pm 0.03$. Both for $x=0.65$ and $x=0.7$ divergent behavior in $\Gamma(T)$ holds down to lowest temperatures, whereas for the $x=0.8$ system a saturation is found below $0.3 \mathrm{~K}$, indicative for the crossover towards LFL behavior. Like in $\mathrm{CeNi}_{2} \mathrm{Ge}_{2}$, both specific heat and thermal expansion follow the predictions of the itinerant theory for a 3D AF QCP. To analyze the critical Grüneisen ratio, the noncritical contributions of specific heat and thermal expansion have been subtracted. The observed $1 / T$ divergence (cf. inset (b) of Fig. 4) proves an itinerant AF QCP in this system [32].

We now turn to the anisotropic $\mathrm{CeMIn}_{5}(\mathrm{M}=\mathrm{Rh}, \mathrm{Co}$, Ir) systems which consist of an alternating series of $\mathrm{CeIn}_{3}$ and $\mathrm{MIn}_{2}$ layers stacked subsequently along the $c$ direction. As a result of the layered crystal structure, the Fermi surface displays a strongly 2D character with cylindrical sheets along the $c$-axis [36]. Superconducting transition temperatures of about $2 \mathrm{~K}$ are observed over wide pressure ranges for the tetragonal $\mathrm{CeRhIn}_{5}$ (at $p \geq 1.6 \mathrm{GPa}$ ) and $\mathrm{CeCoIn}_{5}$ (at ambient pressure) $[37,38]$. The ten times higher $T_{c}$ compared to cubic $\mathrm{CeIn}_{3}$ under hydrostatic pressure has been attributed to the layered crystal structure and, relatedly, strongly anisotropic magnetic fluctuations [38]. Indeed the nuclear magnetic relaxation rate $1 / T_{1}$ of $\mathrm{CeCoIn}$ displays a weak $T^{1 / 4}$ dependence in the normal state between 2 and $40 \mathrm{~K}$ which signals strongly anisotropic quantum critical fluctuations [39]. Previous measurements of the electrical resistivity [40], thermal transport [41, 42], as well as specific heat [43], have revealed pronounced NFL behavior near the upper critical field $B_{c 2}$ of SC $(5 \mathrm{~T}$ along the $c$-axis). In particular a divergence of the $A$ coefficient of the electrical resistivity suggests a field-tuned QCP close to $B_{c 2}$. The Wiedemann-Franz law in the limit $T \rightarrow 0$ is violated along the $c$-axis, while it holds in the perpendicular plane, suggesting an anisotropic destruction of the Fermi surface near the QCP [42]. Here, we use thermal expansion measurements to characterize quantum criticality in $\mathrm{CeCoIn}_{5}$ [44]. As shown by the orange squares in Fig. 5, upon cooling from high temperatures the linear expansion coefficient $\alpha(T) / T$ diverges as $T^{-1}$, which is the temperature dependence predicted by the itinerant theory for a $2 \mathrm{D}$ AF QCP. Below $T^{\star} \approx 0.3 \mathrm{~K}$ (cf. arrows in Fig. 5), however, the data deviate from this behavior and follow $\alpha(T) \sim \sqrt{T}$ down to at least $40 \mathrm{mK}$, which perfectly agrees with 3D AF quantum criticality. In this scenario singular magnetic scattering is restricted to certain "hot lines" along the Fermi surface which shrink as $T \rightarrow 0$, incompatible with a Fermi surface destruction. As the field is increased above $5 \mathrm{~T}$, this crossover scale shifts towards higher temperatures, cf. Fig. 6. A dimensional crossover in quantum critical magnetic metals has recently been considered theoretically [45]. In agreement with our observations on layered $\mathrm{CeCoIn}_{5}$, it has been found that the dimensionality increases as criticality is approached as a consequence of the divergence of the correlation length $\xi: 3 \mathrm{D}$ quantum criticality is expected when $\xi$ exceeds the characteristic anisotropy length scale $\xi_{\text {aniso }}$ that captures the explicit or hidden anisotropy of spin fluctuations. Note, that this theory does not take into account effects of a fully frustrated interplane coupling discussed for the Bose-Einstein condensate of spin triplets in the Mott insulator $\mathrm{BaCuSi}_{2} \mathrm{O}_{6}[46]$. 


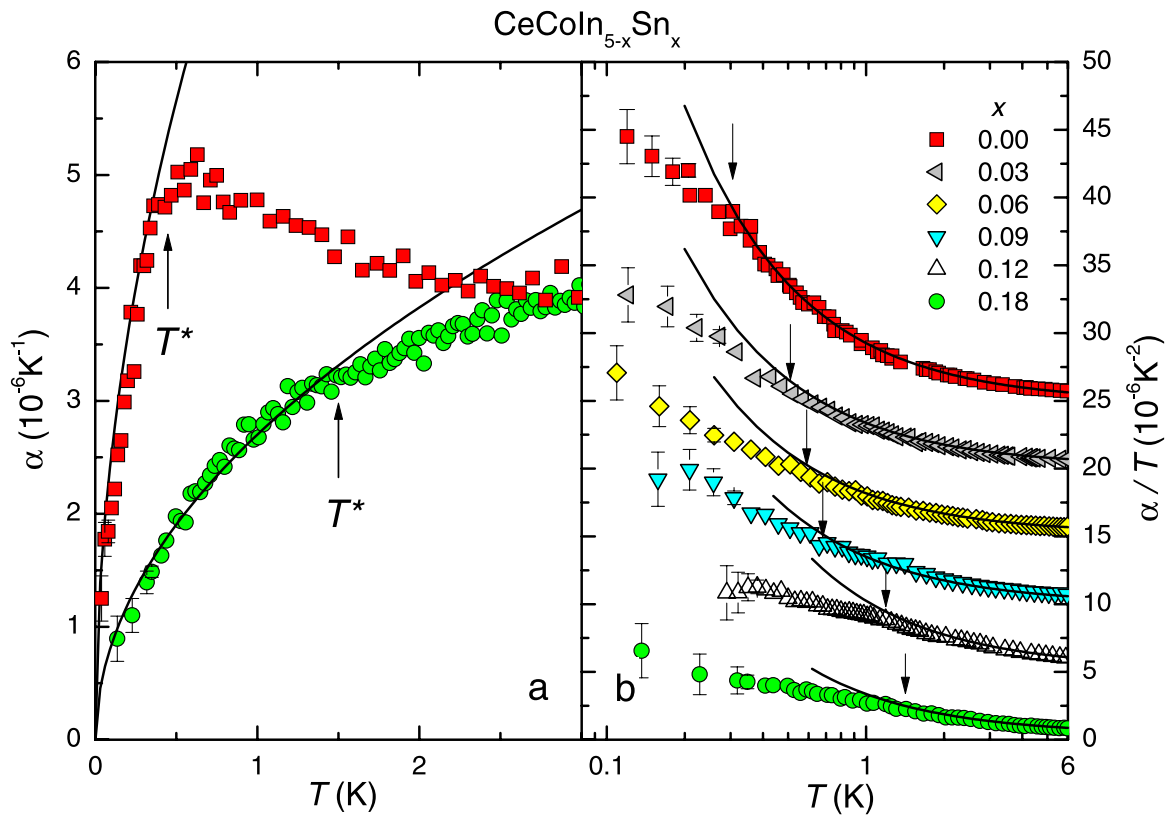

Fig. 5 (Color online) Linear thermal expansion of $\mathrm{CeCoIn}_{5-x} \mathrm{Sn}_{x}$ at magnetic fields $B \| c$ close to the respective upper critical field as $\alpha(T)$ (a) and $\alpha(T) / T$ vs. $T$ on a logarithmic scale (b) [44]. Lines in (a) and (b) display $\alpha \propto \sqrt{T}$ and $\alpha / T \propto T^{-1}$, respectively. Note that the data sets in (b) are shifted by $5 \times 10^{-6} \mathrm{~K}^{-2}$, subsequently

Since lattice disorder enhances isotropic impurity scattering, one would expect a stabilization of 3D behavior by disorder. In order to investigate the influence of disorder on the observed dimensional crossover, the system $\mathrm{CeCoIn}_{5-x} \mathrm{Sn}_{x}$ is best suited. Here the $\mathrm{Sn}$ atoms preferentially substitute within the in-plane In position [47]. The SC transition temperature $T_{c}(x)$ is found to be rapidly suppressed at $x=0.18$, i.e., for $\sim 3.6 \% \mathrm{Sn}$ only. Most remarkably, specific heat and electrical resistivity measurements have been consistent with a QCP located at $B_{c 2}(x)$ for all $x \leq 0.18$ $[48,49]$.

Figure 5 displays respective low-temperature thermal expansion results at $B=$ $B_{c 2}(x)$ with $B=0$ at $x=0.18$. As shown in part (b), the crossover scale $T_{d i m}$ above which 2D quantum critical behavior is found, shifts with increasing Sn doping towards higher temperatures, as anticipated above. Likewise the region of 3D quantum criticality is extended, e.g. for temperatures up to $1.5 \mathrm{~K}$ at $x=0.18$ (cf. the green circles in Fig. 5a). The evolution of the dimensional crossover with Sn doping is displayed in the inset of Fig. 6. The Sn-doping leads to an increase of the residual resistivity from below $1 \mu \Omega \mathrm{cm}$ at $x=0$ to $15 \mu \Omega \mathrm{cm}$ at $x=0.18$ [49]. This moderate disorder effectively stabilizes $3 \mathrm{D}$ quantum criticality. A similar conclusion could also be drawn from our thermal expansion measurements on CeRhIn ${ }_{5-x} \mathrm{Sn}_{x}$ discussed in the following [50].

A comparison of cubic CeIn ${ }_{3-x} \mathrm{Sn}_{x}$ with layered $\mathrm{CeRhIn}{ }_{5-x} \mathrm{Sn}_{x}$, which both show rather similar $T_{\mathrm{N}}(x)$ phase diagrams (cf. Figs. 4 and 7), seems well suited for investigating the influence of lattice anisotropy on quantum criticality. However, it 


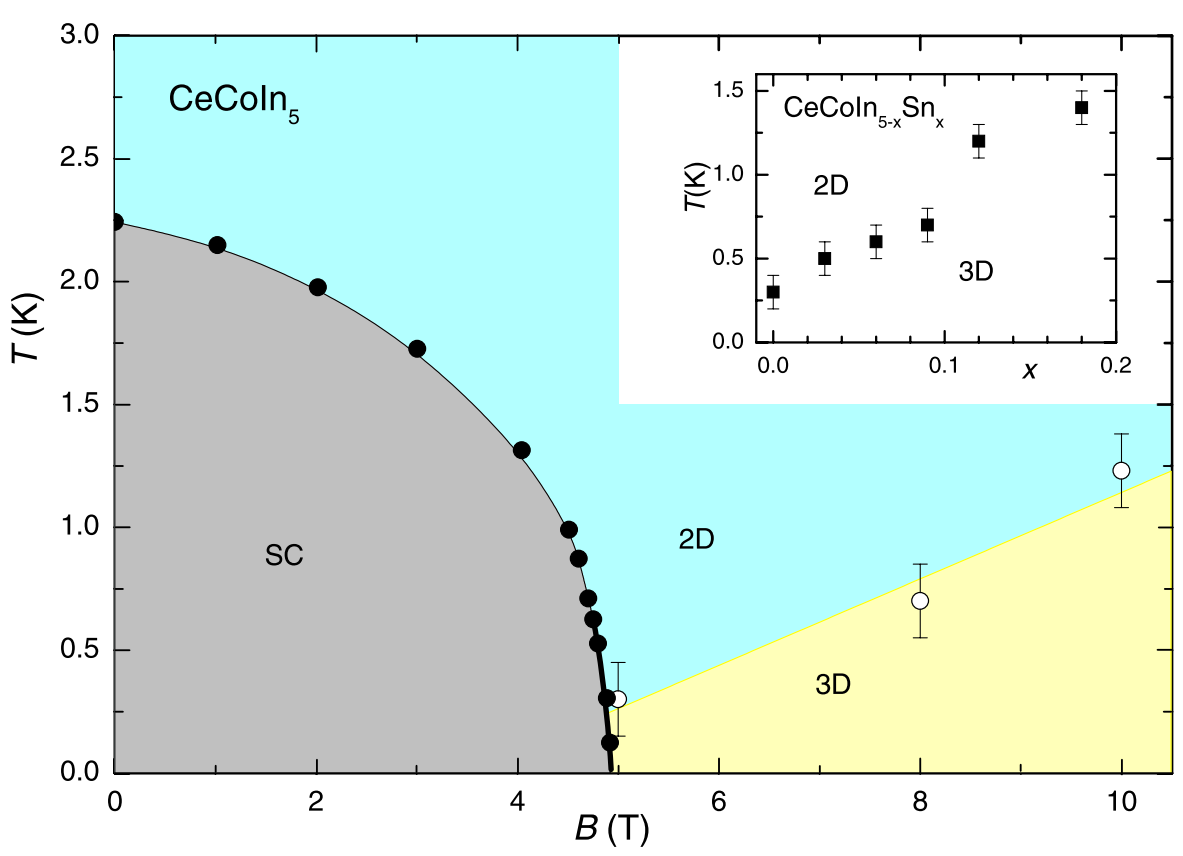

Fig. 6 (Color online) Phase diagram of $\mathrm{CeCoIn}_{5}$ for $B \| c$ as determined from thermal expansion. The SC phase is marked in gray with the first-order boundary below $0.7 \mathrm{~K}$ indicated by the thick black line. Regions where thermal expansion follows 2D- and 3D quantum critical behavior are marked in blue and yellow, respectively. The inset displays the evolution of the crossover with Sn-doping in $\operatorname{CeCoIn}_{5-x} \operatorname{Sn}_{x}$ at the respective $B_{c 2}(x)$ [44]

turned out that, like in $\mathrm{CeCoIn}_{5-x} \mathrm{Sn}_{x}$, disorder tends to stabilize 3D quantum criticality in the layered system. For CeRhIn ${ }_{5-x} \operatorname{Sn}_{x}$ the AF QCP is located at $x_{c} \approx 0.48$, for which the low temperature thermodynamic properties are very similar to those of cubic $\mathrm{CeIn}_{2.35} \mathrm{Sn}_{0.65}$. The linear thermal expansion coefficient follows the predictions of the itinerant theory for a $3 \mathrm{D}$ AF QCP in the entire temperature range up to $6 \mathrm{~K}$ (Fig. 7) [50]. Compared to $\mathrm{CeCoIn}_{4.82} \mathrm{Sn}_{0.18}$ discussed above, the latter system has a four-times higher $\mathrm{Sn}$-concentrations resulting in a three times larger residual resistivity. Whereas $T_{\text {dim }} \approx 1.4 \mathrm{~K}$ in the former case, it is above $6 \mathrm{~K}$ in the latter. These observations fit to the interpretation of impurity scattering smearing effectively out the anisotropy of the quantum critical fluctuations. Nevertheless, an algebraically diverging Grüneisen parameter $\Gamma(T)$ in CeRhIn ${ }_{4.52} \mathrm{Sn}_{0.48}$ proves a truly pressure dependent QCP [19]. This is in contrast to stronger disordered systems in which $\Gamma(T)$ diverges logarithmically $\left(\mathrm{CePd}_{1-x} \mathrm{Rh}_{x}\right)$ [51] or saturates at low-temperatures $\left(\mathrm{Ni}_{x} \mathrm{Pd}_{1-x}\right.$ [52], $\left.\mathrm{URh}_{1-x} \mathrm{Ru}_{x} \mathrm{Ge}[53]\right)$.

\section{Grüneisen Parameter and Magnetocaloric Effect in $\operatorname{YbRh}_{2}\left(\operatorname{Si}_{1-x} G_{x}\right)_{2}$}

We now turn to the tetragonal HF metal $\mathrm{YbRh}_{2} \mathrm{Si}_{2}$, which displays very weak AF ordering at $T_{\mathrm{N}}=70 \mathrm{mK}[29,54]$. This system could be tuned towards a QCP by 


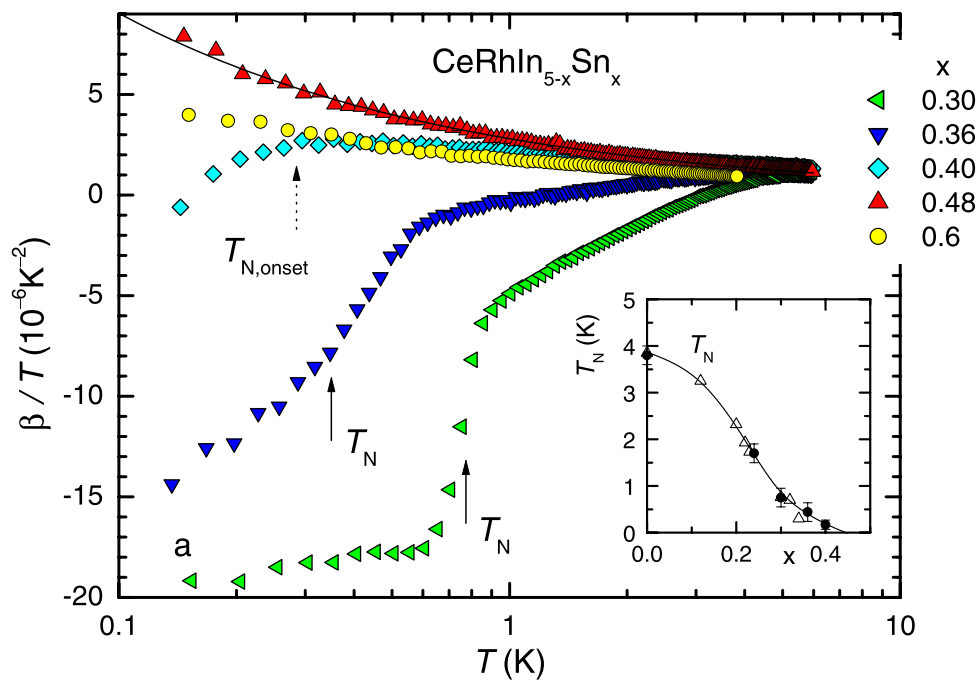

Fig. 7 (Color online) Temperature dependence of the volume thermal expansion coefficient of $\mathrm{CeRhIn}_{5-x} \mathrm{Sn}_{x}$ as $\beta / T$ vs. $T$ on a logarithmic scale (a). Arrows mark Néel temperatures. The line indicates $1 / \sqrt{T}$ dependence. The inset displays the magnetic phase diagram for CeRhIn ${ }_{5-x} \operatorname{Sn}_{x}$ [50]

Fig. 8 (Color online)

Temperature dependence of the Grüneisen parameter of $\mathrm{YbRh}_{2}\left(\mathrm{Si}_{1-x} \mathrm{Ge}_{x}\right)_{2}(x=0$, $0.05)$. The inset displays the critical Grüneisen ratio (after subtraction of normal contributions to thermal expansion) of $\mathrm{YbRh}_{2}\left(\mathrm{Si}_{0.95} \mathrm{Ge}_{0.05}\right)_{2}$. Line indicates $T^{-0.7}$ divergence [21]

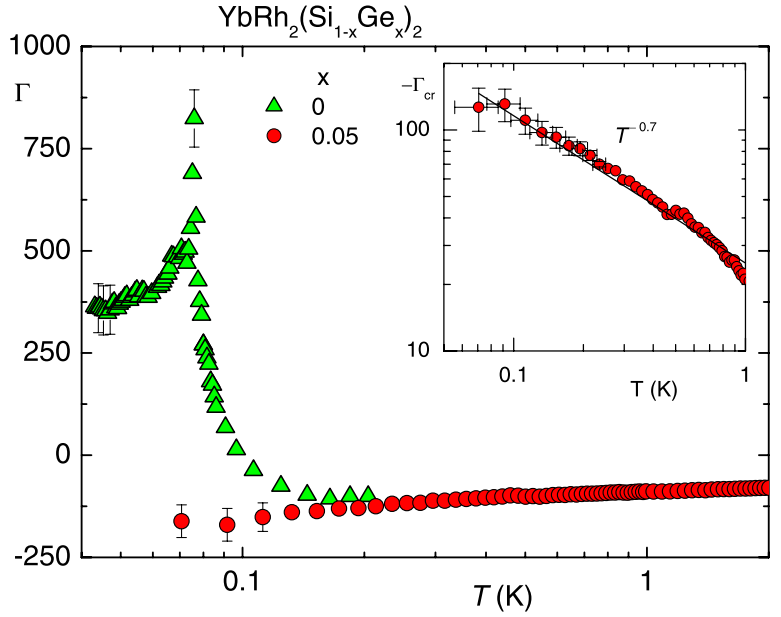

applying a tiny critical magnetic field $B_{c}$ of $0.05 \mathrm{~T}$ or $0.7 \mathrm{~T}$ perpendicular to, or along the $c$-axis, respectively. Alternatively a small negative chemical pressure induced by Ge-doping in $\mathrm{YbRh}_{2}\left(\mathrm{Si}_{1-x} \mathrm{Ge}_{x}\right)_{2}$ with nominal Ge content $x=0.05$ suppresses the ordering to $20 \mathrm{mK}$ [11]. Since the Grüneisen parameter and magnetocaloric effect are most sensitive to pressure and magnetic field tuning, respectively, we focus on the former property for $\mathrm{YbRh}_{2}\left(\mathrm{Si}_{1-x} \mathrm{Ge}_{x}\right)_{2}$ [21] and latter property for the field-tuned $\mathrm{QCP}$ in undoped $\mathrm{YbRh}_{2} \mathrm{Si}_{2}$ [55]. 
Figure 8 shows the Grüneisen parameter $\Gamma(T)$ for $\mathrm{YbRh}_{2}\left(\mathrm{Si}_{1-x} \mathrm{Ge}_{x}\right)_{2}(x=0$ and $x=0.05)[21,52]$. In the paramagnetic regime, $\Gamma<0$, reflecting the negative pressure dependence of the Kondo temperature in Yb-based systems. The AF transition at $x=0$ results in a sign change at $0.1 \mathrm{~K}$ followed by a sharp peak at $T_{\mathrm{N}}$. At temperatures well below $T_{\mathrm{N}}$, LFL behavior has been observed in specific heat and electrical resistivity experiments, with highly enhanced values for the coefficients $\gamma_{0} \approx 1.7 \mathrm{~J} / \mathrm{K}^{2} \mathrm{~mol}$ and $A \approx 22 \mu \Omega \mathrm{cm} / \mathrm{K}^{2}$, respectively [29]. In this regime, the Grüneisen parameter saturates at a giant value of 350 , indicating the huge pressure dependence of the ground state in this system.

The volume expansion induced by the substitution of nominally 5 at\% of Si by the larger but isoelectronic $\mathrm{Ge}$ in $\mathrm{YbRh}_{2}\left(\mathrm{Si}_{0.95} \mathrm{Ge}_{0.05}\right)_{2}$ results in a suppression of the Néel temperature to $T_{\mathrm{N}}=20 \mathrm{mK}$ and critical field to $B_{c}=0.025 \mathrm{~T}$ (within the easy plane $\perp c$ ) [11]. The zero-field volume thermal expansion coefficient of the Ge-doped system, measured at temperatures above $50 \mathrm{mK}$, is negative and identical to that at the critical field [21]. Thus, zero-field data are sufficient to analyze the critical Grüneisen ratio in this temperature regime. The observed fractional exponent in $\Gamma_{\mathrm{cr}} \propto T^{-0.7}$ displayed in the inset of Fig. 8 is incompatible with the itinerant theory for an AF QCP and has been taken as signature for a locally-critical QCP in this system [21]. An extension of the zero-field data for $\mathrm{YbRh}_{2}\left(\mathrm{Si}_{0.95} \mathrm{Ge}_{0.05}\right)_{2}$ to very low temperatures has detected a sign change above $T_{\mathrm{N}}=20 \mathrm{mK}$ in this system as well [56]. Single crystals with larger Ge-content to completely suppress the AF phase at $B=0$ would be highly desirable.

We now turn to the field-induced QCP in undoped $\mathrm{YbRh}_{2} \mathrm{Si}_{2}$ [55]. Isothermal measurements of the Hall coefficient as a function of a tuning field $B \perp c$ have revealed a drastic change at a line $B^{\star}(T)$, resp. $T^{\star}(B)$, which extrapolates to the QCP [30]. These experiments suggest an abrupt change of the Fermi-surface volume at the QCP, supposed to be related to a 4 f-selective Mott transition. Thermodynamic and transport measurements such as magnetization, magnetostriction, magnetoresistance all revealed related crossovers at $B^{\star}(T)$ whose full widths at half maxima vanish in the $T \rightarrow 0$ limit [31]. The temperature derivative of the magnetization, $d M / d T$, which equals the field derivative of the entropy, also peaks at $B^{\star}(T)$, indicating a drastic reduction of spin entropy upon entering the Kondo screened large Fermi volume regime [55]. Here, we focus on the magnetic Grüneisen ratio that is obtained by calculating the ratio of the temperature derivative of the magnetization to the specific heat [55]. In Fig. 9, $-(d M / d T) / T$ is compared with $C / T$. The former property is far more divergent on the approach of the QCP than the latter while both consistently indicate the crossover to LFL behavior at low temperatures for $B>B_{c}$.

The magnetic Grüneisen ratio is analyzed in Fig. 10. The main part displays divergent behavior in the temperature dependence of $\Gamma_{\text {mag }}$ at different magnetic fields $B \geq B_{c}$. Close to the critical field the exponent in $\Gamma_{\text {mag }}(T)$ changes from -2 at high $T$ towards -0.7 at low temperatures. The origin of this crossover is discussed below. Interestingly at low temperatures the exponent of the magnetic Grüneisen ratio equals that of the (thermal) critical Grüneisen parameter $\Gamma_{c r}(T)$. Consequently, the pressure derivative of the critical field, $d B_{c} / d p$, saturates at low temperatures [57]. At $B>B_{c}$, LFL behavior is found in the magnetization and specific heat at low temperatures (cf. Fig. 9), leading to $\Gamma(B)_{T \rightarrow 0}$ which as displayed in the inset of 


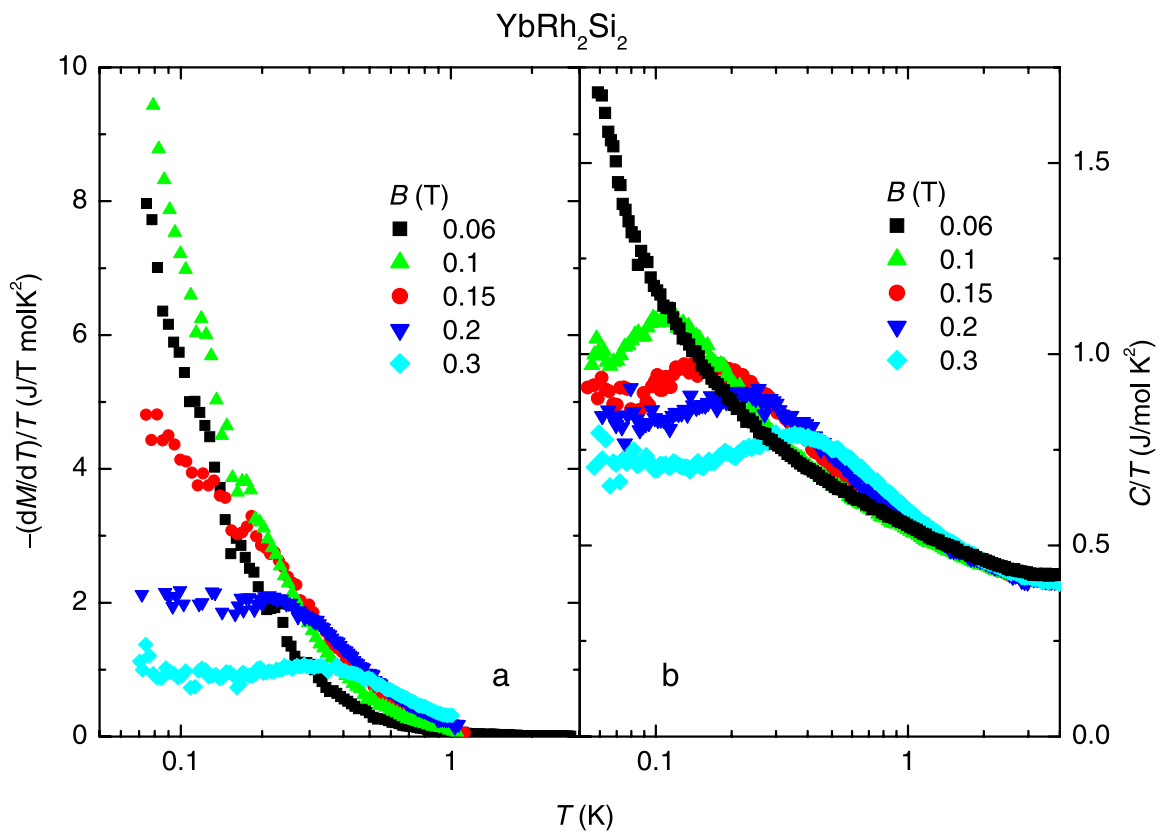

Fig. 9 (Color online) Temperature derivative of the magnetization as $-(d M / d T) / T$ vs. $T$ (a) and electronic specific heat as $C(T) / T$ vs. $T$ (b) for $\mathrm{YbRh}_{2} \mathrm{Si}_{2}$ at various different magnetic fields applied in the easy-plane perpendicular to the $c$-axis [55]

Fig. 10 diverges on the approach of the critical field. The itinerant theory predicts $\Gamma_{\text {mag }}(T=0, B)=-G_{r}\left(B-B_{c}\right)^{-1}$ with $G_{r}=v(d-z)$ where the latter parameter also appears as exponent in the field-dependence of the Sommerfeld coefficient of the specific heat, $\gamma(T=0, B)=\left(B-B_{c}\right)^{G_{r}}$ [19]. As shown in the inset of Fig. 10, the low-temperature saturation values of $\Gamma_{\text {mag }}(B)$ indeed follow such a functional dependence with $G_{r}=-0.3 \pm 0.01$, also consistent with the critical exponent in the divergence of the Sommerfeld coefficient [11]. Since within the itinerant theory $v=1 / 2$ the measured $G_{r}$ is incompatible with any integer values of $d$ and $z$. On the other hand, a recently proposed critical Fermi surface model which may be relevant for a Kondo breakdown QCP discusses electronic criticality with $v=2 / 3, z=3 / 2$ and $d=1$ [58], yielding $G_{r}=-1 / 3$ as found in $\mathrm{YbRh}_{2} \mathrm{Si}_{2}$.

In Fig. 11 the evolution of the magnetic Grüneisen ratio exponent $\varepsilon$, derived from $\Gamma_{\text {mag }} \propto T^{-\varepsilon}$, in the temperature-field phase diagram of $\mathrm{YbRh}_{2} \mathrm{Si}_{2}$ is displayed. The different symbols indicate the position of the crossover scale $T^{\star}(B)$ observed in measurements of the Hall coefficient [30], magnetization [55], susceptibility, magnetostriction and magnetoresistance [31]. Within the LFL regime, the magnetic Grüneisen ratio exponent $\varepsilon \approx 0$, visualized by the violet color code. Upon increasing the temperature, $\varepsilon$ increases. There is a broad regime, in which $\varepsilon \approx 1$ located in the vicinity of $T^{\star}(B)$, whereas the yellow-red regions for $T \geq T^{\star}$ are those where the magnetic Grüneisen exponent is unusually large. Note, that the scaling approach within the itinerant theory predicts $\varepsilon=1 / \nu z$ yielding $\varepsilon=1$ for the AF case. Remark- 


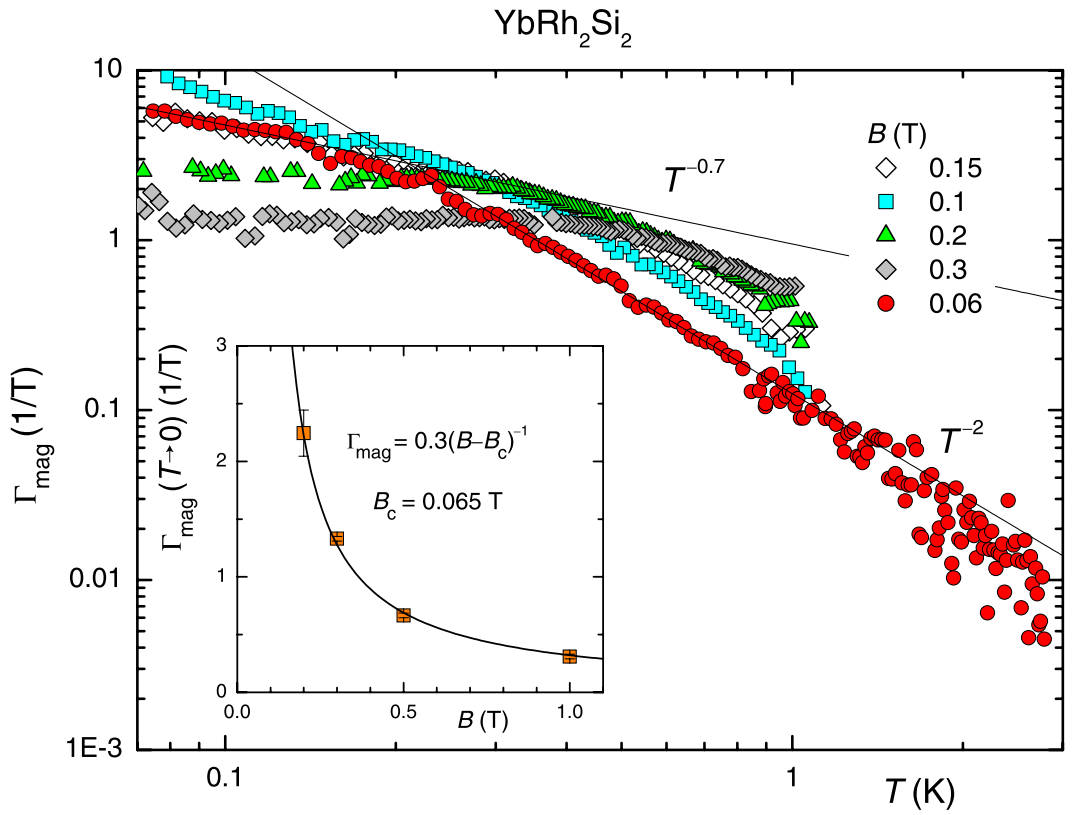

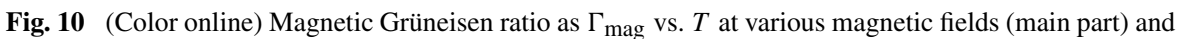
$\Gamma_{\text {mag }}$ vs. $B$ in the field-induced Landau-Fermi-liquid regime (inset) [55]

Fig. 11 (Color online) Contour $\varepsilon$ of $\Gamma_{\text {mag }} \propto T^{-\varepsilon}$ in the temperature vs. magnetic field plane for $\mathrm{YbRh}_{2} \mathrm{Si}_{2}(B \perp c)$. The symbols indicate signatures of $T^{\star}(B)$ in various different properties [57], whereas the black and red lines represent the boundaries of the AF state and the crossover towards Landau-Fermi-liquid behavior in the electrical resistivity, respectively plot for the power-law exponent

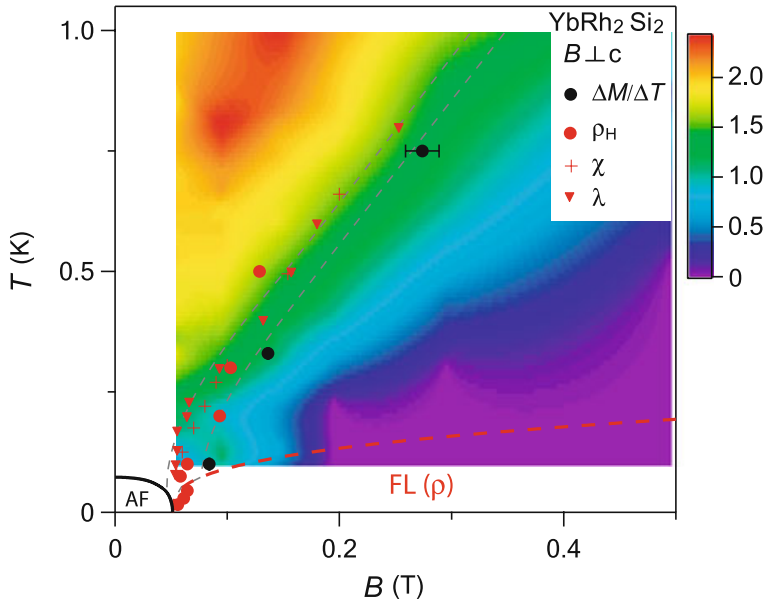

ably, this temperature dependence is found upon approaching the QCP in the vicinity of $T^{\star}(B)$. Unfortunately, we could not yet investigate the magnetic Grüneisen close to $B=0$ or upon crossing the AF phase boundary. Direct measurements of the magnetocaloric effect down to very small $T$ and $B$ are under way [26]. 


\section{Summary and Outlook}

The accumulation of entropy due to the strong quantum fluctuations near a QCP gives rise to singular behavior in the thermal expansion and a divergence of the Grüneisen parameter at any pressure sensitive QCP. Likewise the magnetic Grüneisen ratio which equals the magnetocaloric effect diverges at a field-tuned QCP. Following the first proposal to use these thermodynamic properties to characterize QCPs [19], many different systems have been studied experimentally. Besides the AF QCPs in HF systems discussed in this paper, the above techniques have been applied to study ferromagnetic instabilities in $5 \mathrm{f}-$ and $3 \mathrm{~d}$ based materials. For doped $\mathrm{URh}_{1-x} \mathrm{Ru}_{x} \mathrm{Ge}$ [53] the Grüneisen parameter does not diverge despite a continuous suppression of FM ordering. In clean $\mathrm{URu}_{2} \mathrm{Si}_{2}$ the field-induced suppression of the "hidden-order" phase has been investigated by measurements of the magnetocaloric effect [59]. Signatures of several subsequent first-order quantum phase transitions are observed. In case of the bilayer strontium ruthenate $\mathrm{Sr}_{3} \mathrm{Ru}_{2} \mathrm{O}_{7}$, itinerant metamagnetism leads to a QCP at $8 \mathrm{~T}$ for fields applied along the tetragonal $c$-axis. Magnetostriction [60], thermal expansion [61], as well as measurements of the magnetocaloric effect [62] have revealed a bounded regime, which displays signatures of electronic nematicity in the electrical resistivity and encloses the QCP [63]. Away from this phase, thermal expansion almost perfectly follows the predictions of the itinerant theory for a $z=3$ metamagnetic QCP over several decades in $T /\left(B-B_{c}\right)$ [61]. For the related metamagnetic ruthenate $\mathrm{Ca}_{2-x} \mathrm{Sr}_{x} \mathrm{RuO}_{4}$, less good agreement with the scaling predictions is found, probably due to the larger disorder in this doped system [64]. The thermal expansion behavior close to the metamagnetic crossover in $\mathrm{CeRu}_{2} \mathrm{Si}_{2}$ also displays signatures of quantum critical fluctuations. However, below $0.3 \mathrm{~K}$ a crossover to LFL behavior has been found even at the critical field, indicating that the QCP could not be reached in this system $[65,66]$. The absence of quantum criticality has also been proven for ferromagnetic MnSi under hydrostatic pressure by high-resolution thermal expansion measurements utilizing neutron Larmor diffraction [67]. QCPs in insulators that arise by the closing of a spin-gap have attracted much interest in recent years, due to the observation of a Bose-Einstein condensation of magnons [68]. For example, low-temperature thermal expansion measurements on the two-leg spin ladder $\left(\mathrm{C}_{5} \mathrm{H}_{12} \mathrm{~N}\right)_{2} \mathrm{CuBr}_{4}$ have shown very strong signatures of quantum criticality with a divergence of $\alpha(T)$ in full agreement with quantum Monte Carlo simulations [69].

The focus of this paper has been on Grüneisen parameter studies on QCPs in AF $\mathrm{HF}$ metals. For $\mathrm{CeNi}_{2} \mathrm{Ge}_{2}$ it has been demonstrated, how the critical contributions to thermal expansion and specific heat are determined for the analysis of the critical Grüneisen ratio $\Gamma_{\mathrm{cr}} \propto \beta_{\mathrm{cr}} / C_{\mathrm{cr}}$. The observed $T^{-1}$ dependence is in perfect agreement with the predictions of the itinerant theory. Similar behavior is found for $\mathrm{CeIn}_{3-x} \mathrm{Sn}_{x}$ at $x=0.65$. On the other hand, for $\mathrm{YbRh}_{2}\left(\mathrm{Si}_{0.95} \mathrm{Ge}_{0.05}\right)_{2}$ and $\mathrm{CeCu}_{5.8} \mathrm{Ag}_{0.2}$ disparate behavior has been found which is incompatible with the itinerant theory [21, 27]. Since INS for $\mathrm{CeCu}_{6-x} \mathrm{Au}_{x}$ has proven quasi $2 \mathrm{D}$ magnetic fluctuations [28] and for $\mathrm{YbRh}_{2} \mathrm{Si}_{2}$ a complex interplay of $\mathrm{AF}$ and $\mathrm{FM}$ fluctuations was observed [70], it is important to study the influence of magnetic frustration on quantum criticality. In layered $\mathrm{CeCoIn}_{5-x} \mathrm{Sn}_{x}$ [44], we have discovered a dimensional crossover of quantum criticality, separating $2 \mathrm{D}$ behavior at higher from $3 \mathrm{D}$ behavior at lower temperatures. 
Furthermore, we have proven that this crossover increases with increasing Sn-doping due to the enhancement of isotropic impurity scattering which tends to smear out the magnetic anisotropy. Already moderate disorder could have a strong influence on quantum criticality. This is supported by a comparison of layered CeRhIn ${ }_{4.52} \operatorname{Sn}_{0.48}$ with cubic CeIn $2.35 \mathrm{Sn}_{0.65}$. Remarkably, both systems show very similar thermal expansion behavior near their QCPs, which in both cases is compatible with the 3D SDW scenario [50]. Although the residual resistivity of the former system is not too large $(28 \mu \Omega \mathrm{cm})$, the anisotropy of the magnetic fluctuations is completely suppressed. It is thus desired to investigate quantum criticality in clean stoichiometric systems, which could be tuned by pressure or magnetic field. Here the magnetic Grüneisen ratio and magnetocaloric effect are suitable thermodynamic probes.

As demonstrated in Sect. 3 for the prototypical quantum critical HF metal $\mathrm{YbRh}_{2} \mathrm{Si}_{2}$, the magnetic Grüneisen ratio could provide important information on the nature of the underlying QCP. The observed divergence according to $\Gamma_{\operatorname{mag}}(T \rightarrow$ $0)=-G_{r}\left(B-B_{c}\right)^{-1}$ with $G_{r}=-0.3 \pm 0.01$ sets strong constraints for the scaling parameters $v, d$ and $z$ in this system. We have also discussed a contour plot of the Grüneisen exponent in the $T-B$ phase diagram. In addition to the boundaries of the AF phase and the LFL regime, this phase diagram includes a crossover line $T^{\star}(B)$, which has been associated with a drastic change of the Fermi surface volume due to the destruction of Kondo singlet formation at the QCP. Surprisingly, $T^{\star}(B)$ could be separated from the AF QCP by slight tuning of the unit-cell volume in $\mathrm{Yb}\left(\mathrm{Rh}_{1-x} \mathrm{M}_{x}\right)_{2} \mathrm{Si}_{2}(\mathrm{M}=\mathrm{Co}, \mathrm{Ir})$ [71]. Co is isoelectronic but smaller than $\mathrm{Rh}$ and induces chemical pressure which stabilizes the AF ordering (magnetization results on the Co-doped system agree well with ones of undoped $\mathrm{YbRh}_{2} \mathrm{Si}_{2}$ under hydrostatic pressure) [72], whereas Ir enhances the unit-cell volume and thereby weakens the AF ordered regime $T_{\mathrm{N}}(B)$ similar as in the previously discussed Ge-doped case. In both cases, $T^{\star}(B)$ remains virtually constant, whereas $T_{\mathrm{N}}(B)$ varies by an order of magnitude, resulting in a crossing of $T^{\star}(B)$ and $T_{\mathrm{N}}(B)$ for the Co-doped case (i.e. $\left.B_{c}>B^{\star}\right)$. This suggests the "4f-Mott transition" to be located inside the AF phase, resulting in an itinerant AF QCP [71]. Even more exciting is the case of Ir-doping, where a finite field range $B_{c}<B<B^{\star}$ has been found in which the $4 \mathrm{f}$-moments are suspected to be neither ordered nor Kondo screened and electrical resistivity measurements indicate NFL behavior [71]. This spin-liquid type state, as well as the QCP in the Co-doped system should be further characterized by the Grüeisen parameter and the magnetocaloric effect.

To summarize, thermal expansion, Grüneisen parameter and the magnetocaloric effect are powerful thermodynamic tools to study QCPs. Due to the generic sensitivity of states near QCPs to small changes of pressure or magnetic field, these are most interesting thermodynamic properties. For the future it is expected that they will be used to classify various new types of QCPs in different material classes with electronic correlations.

Acknowledgements Stimulating discussions with M. Brando, J. Custers, M. Garst, N. Oeschler, A. Rosch, J.G. Sereni, Q. Si, M. Vojta and S. Wirth are gratefully acknowledged. We thank O. Stockert, N. Caroca-Canales, O. Tegus and J.A. Mydosh for providing high-quality single crystals and T. Cichorek, K. Neumaier and T. Radu for low-temperature specific heat experiments. Work supported by the DFG through research unit 960 "Quantum phase transitions". 
Open Access This article is distributed under the terms of the Creative Commons Attribution Noncommercial License which permits any noncommercial use, distribution, and reproduction in any medium, provided the original author(s) and source are credited.

\section{References}

1. G.R. Stewart, Rev. Mod. Phys. 56, 755 (1984)

2. H.R. Ott, Characteristic features in heavy electron materials, in Prog. Low Temp. Phys., vol. XI, ed. by D.F. Brewer (Elsevier, Amsterdam, 1987), p. 215

3. N. Grewe, F. Steglich, Heavy fermions, in Handbook on the Physics and Chemistry of Rare Earths, vol. 14, ed. by K.A. Gschneidner Jr., L. Eyring (Elsevier, Amsterdam, 1991), p. 343

4. H.v. Löhneysen, A. Rosch, M. Vojta, P. Wölfle, Rev. Mod. Phys. 79, 1015 (2007)

5. P. Gegenwart, Q. Si, F. Steglich, Nat. Phys. 4, 186 (2008)

6. P. Coleman, C. Pépin, Q. Si, R. Ramazashvili, J. Phys., Condens. Matter 13, R723 (2001)

7. J.A. Hertz, Phys. Rev. B 14, 1165 (1976)

8. A.J. Millis, Phys. Rev. B 48, 7183 (1993)

9. T. Moriya, T. Takimoto, J. Phys. Soc. Jpn. 64, 960 (1995)

10. A. Schröder, G. Aeppli, R. Coldea, M. Adams, O. Stockert, H.v. Löhneysen, E. Bucher, R. Ramazashvili, P. Coleman, Nature 407, 351 (2000)

11. J. Custers, P. Gegenwart, H. Wilhelm, K. Neumaier, Y. Tokiwa, O. Trovarelli, C. Geibel, F. Steglich, C. Pépin, P. Coleman, Nature 424, 524 (2003)

12. Q. Si, M.S. Rabello, K. Ingersent, J.L. Smith, Nature 413, 804 (2001)

13. T. Senthil, S. Sachdev, M. Vojta, Phys. Rev. B 69, 035111 (2004)

14. I. Paul, C. Pépin, M.R. Norman, Phys. Rev. Lett. 98, 026402 (2007)

15. C. Pépin, Phys. Rev. Lett. 98, 206401 (2007)

16. E. Grüneisen, Ann. Phys. (Leipz.) 331, 211 (1908)

17. R. Takke, M. Niksch, W. Assmus, B. Lüthi, R. Pott, R. Schefzyk, D.K. Wohlleben, Z. Phys. B 44, 33 (1981)

18. J. Flouquet, J.C. Lasjaunias, J. Peyrard, M. Ribault, J. Appl. Phys. 53, 2127 (1982)

19. L. Zhu, M. Garst, A. Rosch, Q. Si, Phys. Rev. Lett. 91, 066404 (2003)

20. S. Kambe, J. Flouquet, P. Lejay, P. Haen, A. de Visser, J. Phys. Condens. Matter 9, 4917 (1997)

21. R. Küchler, N. Oeschler, P. Gegenwart, T. Cichorek, K. Neumaier, O. Tegus, C. Geibel, J.A. Mydosh, F. Steglich, L. Zhu, Q. Si, Phys. Rev. Lett. 91, 066405 (2003)

22. P. Gegenwart, F. Kromer, M. Lang, G. Sparn, C. Geibel, F. Steglich, Phys. Rev. Lett. 82, 1293 (1999)

23. G. Knebel, M. Brando, J. Hemberger, M. Nicklas, W. Trinkl, A. Loidl, Phys. Rev. B 59, 12390 (1999)

24. C. Bergmann, H.S. Jeevan, M. Schubert, C. Geibel, P. Gegenwart, Phys. Status Solidi B 247, 694 (2010)

25. C. Bergmann, H.S. Jeevan, Y. Tokiwa, P. Gegenwart, unpublished results

26. Y. Tokiwa et al., to be published

27. R. Küchler, P. Gegenwart, K. Heuser, E.-W. Scheidt, G.R. Stewart, F. Steglich, Phys. Rev. Lett. 93, 096402 (2004)

28. O. Stockert, H.v. Löhneysen, A. Rosch, N. Pyka, M. Loewenhaupt, Phys. Rev. Lett. 80, 5627 (1998)

29. P. Gegenwart, J. Custers, C. Geibel, K. Neumaier, T. Tayama, K. Tenya, O. Trovarelli, F. Steglich, Phys. Rev. Lett. 89, 056402 (2002)

30. S. Paschen, T. Lühmann, S. Wirth, P. Gegenwart, O. Trovarelli, C. Geibel, F. Steglich, P. Coleman, Q. Si, Nature 432, 881 (2004)

31. P. Gegenwart, T. Westerkamp, C. Krellner, Y. Tokiwa, S. Paschen, C. Geibel, F. Steglich, E. Abrahams, Q. Si, Science 315, 969 (2007)

32. R. Küchler, P. Gegenwart, J. Custers, O. Stockert, N. Caroca-Canales, C. Geibel, J.G. Sereni, F. Steglich, Phys. Rev. Lett. 96, 256403 (2006)

33. N.D. Mathur, F.M. Grosche, S.R. Julian, I.R. Walker, D.M. Freye, R.K.W. Haselwimmer, G.G. Lonzarich, Nature 394, 39 (1998)

34. P. Pedrazzini, M. Gómez Berisso, N. Caroca-Canales, M. Deppe, C. Geibel, J.G. Sereni, Eur. Phys. J. B 38, 445 (2004)

35. M. Garst, A. Rosch, Phys. Rev. B 72, 205129 (2005)

36. R. Settai, H. Shishido, S. Ikeda, Y. Murakawa, M. Nakashima, D. Aoki, Y. Haga, H. Harima, Y. Ônuki, J. Phys. Condens. Matter 13, L627 (2001) 
37. H. Hegger, C. Petrovic, E.G. Moshopoulou, M.F. Hundley, J.L. Sarrao, Z. Fisk, J.D. Thompson, Phys. Rev. Lett. 84, 4986 (2000)

38. C. Petrovic, P.G. Pagliuso, M.F. Hundley, R. Movshovic, J.L. Sarrao, J.D. Thompson, Z. Fisk, P. Monthoux, J. Phys. Condens. Matter 13, L337 (2001)

39. Y. Kawasaki, S. Kawasaki, M. Yashima, T. Mito, G.-q. Zheng, Y. Kitaoka, H. Shishido, R. Settai, Y. Haga, Y. Ônuki, J. Phys. Soc. Jpn. 72, 2308 (2003)

40. J. Paglione, M.A. Tanatar, D.G. Hawthorn, E. Boaknin, R.W. Hill, F. Ronning, M. Sutherland, L. Taillefer, C. Petrovic, P.C. Canfield, Phys. Rev. Lett. 91, 246405 (2003)

41. J. Paglione, M.A. Tanatar, D.G. Hawthorn, F. Ronning, R.W. Hill, M. Sutherland, L. Taillefer, C. Petrovic, Phys. Rev. Lett. 97, 106606 (2006)

42. M.A. Tanatar, J. Paglione, C. Petrovich, L. Taillefer, Science 316, 1320 (2007)

43. A. Bianchi, R. Movshovich, I. Vekhter, P.G. Pagliuso, J.L. Sarrao, Phys. Rev. Lett. 91, 257001 (2003)

44. J.G. Donath, F. Steglich, E.D. Bauer, J.L. Sarrao, P. Gegenwart, Phys. Rev. Lett. 100, 136401 (2008)

45. M. Garst, L. Fritz, A. Rosch, M. Vojta, Phys. Rev. B 78, 235118 (2008)

46. S.E. Sebastian, N. Harrison, C.D. Batista, L. Balicas, M. Jaime, P.A. Sharma, N. Kawashima, I.R. Fisher, Science 441, 617 (2006)

47. M. Daniel, E.D. Bauer, S.-W. Han, C.H. Booth, A.L. Cornelius, P.G. Pagliuso, J.L. Sarrao, Phys. Rev. Lett. 95, 016406 (2005)

48. E.D. Bauer, C. Capan, F. Ronning, R. Movshovic, J.D. Thompson, J.L. Sarrao, Phys. Rev. Lett. 94, 047001 (2005)

49. E.D. Bauer, F. Ronning, C. Capan, M.J. Graf, D. Vandervelde, H.Q. Yuan, M.B. Salamon, D.J. Mixson, N.O. Moreno, S.R. Brown, J.D. Thompson, R. Movshovic, M.F. Hundley, J.L. Sarrao, P.G. Pagliuso, S.M. Kauzlarich, Phys. Rev. B 73, 245109 (2006)

50. J.G. Donath, F. Steglich, E.D. Bauer, F. Ronning, J.L. Sarrao, P. Gegenwart, Eur. Phys. Lett. 87, 57011 (2009)

51. T. Westerkamp, M. Deppe, R. Küchler, M. Brando, C. Geibel, P. Gegenwart, A.P. Pikul, F. Steglich, Phys. Rev. Lett. 102, 206404 (2009)

52. R. Küchler, P. Gegenwart, F. Weickert, N. Oeschler, T. Cichorek, M. Nicklas, N. Carocca-Canales, C. Geibel, F. Steglich, Physica B 378-380, 36 (2006)

53. N.T. Huy, A. Gasparini, J.C.P. Klaasse, A. de Visser, S. Sakarya, N.H. van Dijk, Phys. Rev. B 75, 212405 (2007)

54. O. Trovarelli, C. Geibel, S. Mederle, C. Langhammer, F.M. Grosche, P. Gegenwart, M. Lang, G. Sparn, F. Steglich, Phys. Rev. Lett. 85, 626 (2000)

55. Y. Tokiwa, T. Radu, C. Geibel, F. Steglich, P. Gegenwart, Phys. Rev. Lett. 102, 066401 (2009)

56. R. Küchler, F. Weickert, P. Gegenwart, N. Oeschler, J. Ferstl, C. Geibel, F. Steglich, J. Magn. Magn. Mater. 272-276, 229 (2004)

57. Y. Tokiwa, C. Geibel, F. Steglich, P. Gegenwart, J. Phys., Conf. Ser. 200, 012205 (2010)

58. T. Senthil, Phys. Rev. B 78, 035103 (2008)

59. M. Jaime, K.H. Kim, G. Jorge, S. McCall, J.A. Mydosh, Phys. Rev. Lett. 89, 287201 (2002)

60. S.A. Grigera, P. Gegenwart, R.A. Borzi, F. Weickert, A.J. Schofield, R.S. Perry, T. Tayama, T. Sakakibara, Y. Maeno, A.G. Green, A.P. Mackenzie, Science 306, 1154 (2004)

61. P. Gegenwart, F. Weickert, M. Garst, R.S. Perry, Y. Maeno, Phys. Rev. Lett. 96, 136402 (2006)

62. A.W. Rost, R.S. Perry, J.-F. Mercure, A.P. Mackenzie, S.A. Grigera, Science 325, 1360 (2009)

63. R.A. Borzi, S.A. Grigera, J. Farrell, R.S. Perry, S.J.S. Lister, S.L. Lee, D.A. Tennant, Y. Maeno, A.P. Mackenzie, Science 315, 214 (2007)

64. J. Baier, P. Steffens, O. Schumann, M. Kriener, S. Stark, H. Hartmann, O. Friedt, A. Revcolovschi, P.G. Radaelli, S. Nakatsuji, Y. Maeno, J.A. Mydosh, T. Lorenz, M. Braden, J. Low Temp. Phys. 147, 405 (2007)

65. F. Weickert, P. Gegenwart, J.A. Mydosh, F. Steglich, C. Kanadani, Y. Tabata, T. Taniguchi, S. Kawarazaki, Physica B 359, 68 (2005)

66. F. Weickert, M. Brando, F. Steglich, P. Gegenwart, M. Garst, Phys. Rev. B 81, 134438 (2010)

67. C. Pfleiderer, P. Böni, T. Keller, U.K. Rößler, A. Rosch, Science 316, 1871 (2007)

68. T. Giamarchi, C. Rüegg, O. Tchernyshyov, Nat. Phys. 4, 198 (2008)

69. T. Lorenz, O. Heyer, M. Garst, F. Anfuso, A. Rosch, C. Rüegg, K. Krämer, Phys. Rev. Lett. 100, 067208 (2008)

70. P. Gegenwart, J. Custers, Y. Tokiwa, C. Geibel, F. Steglich, Phys. Rev. Lett. 94, 076402 (2005)

71. S. Friedemann, T. Westerkamp, M. Brando, N. Oeschler, S. Wirth, P. Gegenwart, C. Krellner, C. Geibel, F. Steglich, Nat. Phys. 5, 465 (2009)

72. Y. Tokiwa, P. Gegenwart, C. Geibel, F. Steglich, J. Phys. Soc. Jpn. 78, 123708 (2009) 